\title{
A DIFUSÃO DE SISTEMAS DE VIDEOVIGILÂNCIA NA URBE CONTEMPORÂNEA: UM ESTUDO INSPIRADO EM ARGOS PANOPTES, CÉREBROS ELETRÔNICOS E SUAS CONEXÕES COM A LIBERDADE E A IGUALDADE*
}

\author{
THE SPREAD OF VIDEOSURVAILLANCE SYSTEMS IN CONTEMPO- \\ RARY CITIES: A STUDY INSPIRED BY ARGOS PANOPTES, ELETRONIC \\ BRAINS AND ITS CONNECTIONS TO LIBERTY AND EQUALITY
}

MARCOS CATALAN

\begin{abstract}
RESUMO
As cidades são construções sociais que, em muito, transcendem quaisquer tentativas de pensá-las como espaços inertes. Espalhados, através delas, sistemas de videovigilância utilizados, inicialmente, no controle do tráfego de veículos, hodiernamente, carregam consigo promessas de segurança, desprezando a violação de direitos delineados a partir das ideias de liberdade positiva e de igualdade substancial. Desde essa hipótese, o artigo foi esboçado em quatro partes. Inicialmente, rabiscando intersecções possíveis entre as cidades, o medo, a vigilância e o Direito. Em um segundo momento, permitindo que aflorem aspectos relevantes da pesquisa empírica realizada buscando mapear informações atadas às preocupações que envolvem a instalação e operação das redes de videovigilância em duas importantes cidades brasileiras. O terceiro capítulo contém a crítica ao modelo existente fundada no acoplamento de um Direito deveras fragmentado à fundamentalidade que informa os direitos civis. O quarto, enfim, comprova a hipótese. A metodologia eleita transita por
\end{abstract}

\begin{abstract}
Cities are social constructions that cannot be seen as inert spaces. Scattered through them, video surveillance systems initially used to control vehicular traffic, nowadays transmit security promises, ignoring the violation of rights connected to freedom and substantial equality. From this hypothesis, this paper was drafted in four parts. Initially, scribbling possible intersections between cities, fear, vigilance and law. Secondly, allowing the emergence of relevant aspects seen in the empirical research carried out focusing to map installation and operation of video surveillance networks in two important Brazilian cities. The third chapter analyses the data that was obtained reflecting about our Law and the idea of fundamental rights. The last one, finally, proves the hypothesis. The methodology transits through interviews and critical analysis of the state of the art about the theme.
\end{abstract}

KEYWORDS: Urban space. Equality. Freedom. Fear. Consumer society. Video surveillance.

* Este artigo foi construído no desvelar dos projetos de pesquisa Reducing boundaries: understanding exclusion through security defensive systems in wealthy urban [http://www. reducing-boundaries.net/], financiado com recursos do Marie Curie European project [FP7-PEOPLE-2013-IRSES] e A difusão de sistemas de videovigilância e a tutela de direitos densificadores da liberdade e da igualdade no Brasil, que conta com recursos da Universidade La Salle, Canoas, Rio Grande do Sul.

* Pós-doutor pela Facultat de Dret da Universitat de Barcelona. Doutor summa cum laude em Direito pela Faculdade do Largo do São Francisco, Universidade de São Paulo. Mestre em Direito pela Universidade Estadual de Londrina. Professor no Mestrado em Direito e Sociedade da Universidade LaSalle. Professor no curso de Direito da Unisinos. Editor da Revista Eletrônica Direito e Sociedade. Visitor scholar no Istituto Universitario di Architettura di Venezia. E-mail: marcoscatalan@uol.com.br. 
entrevistas e análise crítica do estado da arte na compreensão do tema.

PALAVRAS-CHAVE: Espaço urbano. Igualdade. Liberdade. Medo. Sociedade de consume. Videovigilância.

SUMÁRIO: 1. A CIDADE EM MOVIMENTO: UMA SUCINTA REFLEXÃO A TÍTULO DE INTROITO 2. ABRINDO FISSURAS NA FENOMENOLOGIA DAS RELAÇÕES SOCIAIS: MÉTODO, METODOLOGIA E DADOS RELEVANTES ACERCA DE PORTO ALEGRE E CANOAS 3. A VINGANÇA DE ARGOS PANOPTES 4. CONCLUSÕES INCONCLUSIVAS: SOBRE SOMBRAS E VAGALUMES. REFERENCIAS.

L'evoluzione tecnologica, mentre ci offre straordinarie possibilità, mette anche a forte rischio la nostra sfera personale, la nostra libertà ${ }^{1}$.

\section{A CIDADE EM MOVIMENTO: UMA SUCINTA REFLEXÃO A TÍTULO DE INTROITO}

A urbe é moldada pela mão humana.

Nela, embora o espaço [que ocupa] seja produto e produtor de dinâmicas que regem o seu tempo, a experiência da vida urbana [e] a relação de pertencimento [aos seus espaços] persistem [ou não] em meio a um conjunto de transformações que incidem nas dimensões técnicas e tecnológicas, nos aspectos sociais e ambientais, na desvalorização do espectro de atuação do Estado, na desconfiança nos sistemas institucionais, na concentração de renda e disfunções de atividades, no empobrecimento dos sistemas simbólicos, na polarização social e retração das formas de vida coletiva, [na] instrumentalização dos espaços de ação e [na] redução do valor do público ${ }^{3}$.

1 PAISSAN, Mauro. La privacy è morta, viva la privacy. Milano: Ponte alle Grazie, 2009. p. 7.

2 O estilo literário, conscientemente incorporado aos raciocínios alinhavados no desvelar deste artigo, em alguma medida, busca inspiração na imaginação [jus]sociológica. Tal escolha visa a capacitar homens e mulheres a navegarem nos significados de sua época de modo a nos compreenderem, permitindo a multiplicação das narrativas que chegam até eles, quiçá, como mensagens em garrafas. Seus critérios de validade, portanto, são "narrativos e experimentais" e, também por isso, em inúmeras ocasiões, hão de desbordar os moldes geométricos que ainda parecem informar o senso comum imaginário dos juristas. JACOBSEN, Michael Hviid; TESTER, Keith. Introdução. In BAUMAN, Zygmunt. Para que serve a sociologia? Trad. Carlos Alberto Medeiros. Rio de Janeiro: Zahar, 2015. p. 13-14.

3 ALVES, Manoel Rodrigues; RIZEK, Cibele Saliba. Cidade contemporânea, cidade do empresariamento: aspectos da produção socioespacial do urbano. In RODRIGUEZ, José Exequiel Basini et al (Org.). Cidades, fronteiras e mobilidade humana. Manaus: EDUA, 2014. p. 81. 
Em movimento, portanto, e expostas a forças deveras complexas, as cidades são modeladas, modificadas, reformadas, alteradas, adulteradas e corrompidas sem que os seus artífices tenham como controlar uma parcela significativa dos efeitos gestados em ações que, entremeio aos fluxos e refluxos dos quais emergem ruas e avenidas, becos e vielas, praças, parques e escolas, ginásios e estádios e fábricas, barracos, casas, apartamentos, palacetes ou arranha-céus, nada têm de neutras ou inertes, embora seus habitantes, aparentemente, não o percebam, vendo-as, senão como transformações naturais ${ }^{4}$.

Imersos nesse turbilhão e entremeio à agitação e à transformação em curso em tais espaços marcados pela complexidade, parece impossível desprezar um dos apêndices mais salientes neste incessante processo atado ao avanço da técnica ${ }^{5}$ : a propagação de sistemas (ou) redes de videovigilância. Públicos e (ou) privados - pouco importa aqui -, suas câmeras e mecanismos de transmissão de dados são elementos cada vez mais presentes nas cidades contemporâneas.

Sistemas que, ao prometerem proteção, transformam as cidades em prisões ${ }^{6}$.

$\mathrm{Na}$ urbe contemporânea, troca-se liberdade por segurança ${ }^{7}$ e, ainda assim, seus habitantes - tomados pelo medo ${ }^{8}$ - parecem não se preocupar com o fato de que (a) liberdades existenciais estão sendo barganhadas, cotidianamente, permutadas por promessas, tão abstratas, quanto vazias, de combate à criminalidade e (ou) ao terrorismo e, muito menos, com (b) a constatação de que "seguem a

4 SUÁREZ, Carlo Emilio Piazzini. Los estudios socioespaciales: campo de tensiones y caminos recorridos. In RODRIGUEZ, José Exequiel Basini et al (Org.). Cidades, fronteiras e mobilidade humana. Manaus: EDUA, 2014. p. 22-23. "Las percepciones del espacio físico y las concepciones del espacio, aun cuando no permiten dar cuenta integral de las espacialidades, hacen parte de la producción social del espacio, lo cual se hace visible en ese tercer momento de los espacios vividos. Con ello, se reconocen dos asuntos fundamentales: primero, que las percepciones y concepciones del espacio como una entidad "natural", son en realidad producciones sociales, y segundo, que las elaboraciones discursivas del espacio tienen un límite y que por lo tanto no pueden pretender reemplazar las espacialidades por su representación en el mundo del lenguaje".

5 MASI, Domenico de. A sociedade pós-industrial. 4. ed. Trad. Anna Maria Capovilla et all. São Paulo: Senac, 2003.

6 HUMANES, Mariano Pérez. Fronteras urbanas: el espacio público como espacio de recusión. In RODRIGUEZ, José Exequiel Basini et al (Org.). Cidades, fronteiras e mobilidade humana. Manaus: EDUA, 2014. p. 179. “[...] bajo el pretexto de la seguridad ciudadana, la tendencia general de las administraciones urbanas ha consistido en normalizar y homologar los espacios públicos en un proceso de privatización y control que ha transformado la ciudad contemporánea en una nueva prisión”.

7 BAUMAN, Zygmunt. Para que serve a sociologia? Trad. Carlos Alberto Medeiros. Rio de Janeiro: Zahar, 2015. p. 101. CATALAN, Marcos. Avanço da técnica e afetação da vida privada no espaço público. Revista Direitos Humanos e Democracia, v. 6, p. 455-464, 2018. DÉCARLI, Natália. La frontera del miedo global: proteger para ser. In RODRIGUEZ, José Exequiel Basini et al (Org.). Cidades, fronteiras e mobilidade humana. Manaus: EDUA, 2014. p. 158.

8 COSTA, Renata Almeida da. Cultura do medo e espaço urbano: um olhar reflexivo sobra a sensação social de insegurança. In SCHWARTZ, Germano; FERNÁNDEZ, Albert Noguera. (Org.) Cultura e identidade em tempo de transformações. Curitiba: Juruá. 2011. p. 219-238. 
correnteza, obedecendo [...] suas rotinas diárias [...] resignados diante da impossibilidade de mudá-la[s]", aliás, se é que o percebem.

Em tais cenários,

CCTV systems appeared in the 1970s in the European context, initially to assist traffic regulation in congested cities. Later on, it began to be used in private spaces menaced by the supposedly growing criminality, such as banks, shops, and malls. It is to achieve the same goal of improving security of persons and their property that the use of CCTV spread to public spaces, and began to be used by public authorities. The flourishing security market and the incentivizing state policies in the UK at the beginning of the 1990s turned this type of use of CCTV into an unavoidable tool to ensure security in other European cities ${ }^{10}$.

Hodiernamente, como antecipado, indispensável recurso às lentes epistêmicas lapidadas ao largo dos últimos anos permite enxergar, com incomum clareza, a existência de redes de controle formadas na conjunção de (a) câmeras que possuem a função zoom melhorada a cada pequenino giro anunciado pela indústria tecnológica ou, ainda, com potencial cada vez maior para capturar e gravar, com acentuada nitidez ${ }^{11}$, cenas havidas também na calada da noite, (b) ondas e cabos pelos quais mais e mais informações podem transitar sem se perder, ainda que, também, possam vir a alimentar os bancos de dados de hackers e crakers, (c) monitores e telas cada vez maiores, mais numerosos e capazes de reproduzir cenas mais nítidas, mesmo que isso não ocorra, necessariamente, quando do seu uso, (d) melhor capacidade de armazenamento e tratamento dos dados capturados ao atravessarem as quase incomensuráveis pupilas vítreas fundidas a um dos muitos olhos dos anjos incrustrados nos postes $\mathrm{e}(\mathrm{ou})$ nas paredes incorporadas à urbe, (e) profissionais, não necessariamente, bem treinados e (ou) descansados ${ }^{12} \mathrm{e}$, por que não, (f) sistemas de reconhecimento facial e (ou) softwares aptos à leitura das placas dos automóveis que vão e vêm pelas veias que rasgam as cidades, quase

9 BAUMAN, Zygmunt. Para que serve a sociologia? Trad. Carlos Alberto Medeiros. Rio de Janeiro: Zahar, 2015. p. 20.

10 BOURDOUX, Gil. Vidéosurveillance et police. Quel(s) coût(s) pour la police? Quel(s) coût(s) pour la société? In DEVRESSE, Marie-Sophie; PIERET, Julien (Dir.). La vidéosurveillance: entre usages politiques et pratiques policières, Brussels: Politeia, 2009. p. 14-15 apud DEBAILLEUL Corentin; KEERSMAECKER Pauline De. Towards the panoptic city: the proliferation of video surveillance in Brussels and Copenhagen. Thesis (Master) - Université Libre de Bruxelles, Bruxelles, 2014. p. 12.

$11 \mathrm{Na}$ cidade de Canoas, uma das bases geográficas em que essa fase da pesquisa empírica foi realizada, o zoom pode chegar a 250 metros e, consoante informações colhidas, isso implica a possibilidade de ler a placa de um automóvel estacionado a essa distância das câmeras.

12 MElgaÇO, Lucas; VERFAILlie, Kristof; HILDEBRANDT, Mireille. CCTV and Smart CCTV effectiveness: a meta-level analysis. SIAM - Security Impact Assessment Measures. Brussel: Vrije Universiteit Brussel, 2013. p. 20. O professor brasileiro, que leciona em Bruxelas aponta, com lastro em sólida e farta literatura especializada, que a exposição a horas e horas de vídeos, no mais das vezes, desinteressantes, pode tornar a atividade de observá-los deveras entediante. 
sempre, sem feri-las e, ainda, a filtrar comportamentos que podem ser previamente programados como suspeitos ${ }^{13} \mathrm{e}$, portanto, dignos de atenção.

$\mathrm{O}$ incessante labor de Chronos permite inferir, como se pode identificar, que o recurso aos sistemas de videovigilância transcendeu as preocupações havidas quando de sua gênese na década de setenta do século XX - atadas, naquela ocasião, à ordenação do trânsito -, passando a controlar, com imperceptível suavidade e custos não tão palatáveis, o exercício de incomensuráveis liberdades positivas ${ }^{14}$ em muitos dos espaços públicos e privados entranhados à urbe, sem razões que o legitimem, ética e (ou) juridicamente.

$\mathrm{O}$ inconstante fluxo do tempo revela, ainda, como, em pouco mais de quarenta anos, "large CCTV networks get connected to one another, shaping surveillance webs that remain invisible for the citizens" ${ }^{15}$, criando uma rede de controle social armada no interior de densa névoa que dificulta (a) a compreensão de que os sistemas de videovigilância difundidos pelas cidades, cotidianamente, promovem a estigmatização das diferenças existentes entre as pessoas, deixando de valorar, consequentemente, a alteridade e diversos pluralismos, obnubilando a decodificação da incomensurável violência - havida nas esferas real e simbólica - imantada a cada aresta sua e às chagas que por elas são abertas nas telas sobre as quais o princípio constitucional da isonomia ${ }^{16}$ deveria ser colorido, dia após dia, no universo das relações sociais.

Há de registrar-se, ademais, que a expansão das redes de videovigilância parece ter sido legitimada pelo silêncio - mas, também, outras vezes, pelo clamor $^{17}$ - de pessoas nocauteadas pelo zunir midiático de promessas de combate à criminalidade ${ }^{18} \mathrm{ou}$, paralelamente, pelas muitas cenas de manifesto desprezo à

13 Idem. p. 20. "Detection, tracking and classification of targets are the main tasks expected from video analytics engines. Detection refers to the identification of what physical objects exist in the surveillance area, tracking is the understanding of how they move and classification is related to the labelling of objects as human, vehicle, animal and to the interpretation as normal or abnormal objects or behaviours".

14 Constituição Federal brasileira. Art. $5^{\circ}$ Todos são iguais perante a lei [...] garantindo-se aos brasileiros e aos estrangeiros residentes no País a inviolabilidade do direito à vida, à liberdade, à igualdade, à segurança e à propriedade [...] XXXII - o Estado promoverá, na forma da lei, a defesa do consumidor.

15 HEMPEL, Leon; TÖPFER, Eric. CCTV in Europe. Final Report. UrbanEye project: on the threshold to urban Panopticon? Analysing the employment of CCTV in European cities and assessing its social and political impacts, Berlin: Centre for technology and society of the Technical University Berlin, 2004. p. 3-68. Disponível em: em <www.urbaneye.net/results/results. htm>. Acesso em 19.jan.2016.

16 GUEDES, Jefferson Carús. Dimensões linguísticas da desigualdade no Brasil: os diversos nomes legais de um mesmo fenômeno. Revista Brasileira de Políticas Públicas, v. 5, p. 59-76, 2015.

17 A pesquisa empírica realizada em Canoas revelou que os cidadãos postulam a instalação de câmeras perante o Município.

18 DEBAILLEUL Corentin; KEERSMAECKER Pauline De. Towards the panoptic city: the proliferation of video surveillance in Brussels and Copenhagen. Thesis (Master) - Université Libre 
vida que transitam pelos televisores brasileiros como se fossem filmes publicitários de gosto duvidoso, mesmo quando "os dados que [trafegam] na sociedade da informação têm um conjunto de caracteres capazes de identificar aspectos imanentes [...] as suas relações afetivas, familiares, patrimoniais, registros telefônicos, convicções políticas e sexuais”, aspectos, como se observa, não apenas atados as suas existências cotidianas, como, igualmente, afetos à privacidade ${ }^{19} \mathrm{e}[\mathrm{ou}]$ à intimidade, portanto, afetos à seara dos direitos de personalidade.

$\mathrm{E}$, ainda que muitos não o notem, o Direito também atua nesse processo de construção e reconfiguração caótica de espaços públicos e privados, cada vez mais fragmentados, nas cidades contemporâneas. Age, ademais, ao tutelar ainda que, muitas vezes, sem efetividade perceptível - o exercício de liberdades positivas, a promoção da igualdade substancial e a experimentação cotidiana dos diretos de personalidade, eis que cabe a ele imputar - para restringir a reflexão à esfera do direito civil - sanções inibitórias, demolitórias ou impor a reparação dos danos havidos na seara fenomênica. Enfim, não se pode olvidar, também, que a ele cabe, enquanto práxis imantada às vidas vividas na urbe, autorizar e proibir projetos arquitetônicos e (ou) de engenharia, liberar construções, emitir licenças e permissões, ordenar demolições, modular, regrar e disciplinar condutas.

O Direito ${ }^{20}$, aliás, ao legitimar essa manifesta mutação $0^{21}$, também parece não notar, não perceber ou não entender a disseminação de parte das ofensas que ele acaba por sofrer, ao mesmo tempo em que, talvez, igualmente, não parece capaz de identificar que, ao manter-se inerte, se nega a cumprir sua principal razão de ser: servir a pessoa humana, toda pessoa humana.

\section{ABRINDO FISSURAS NA FENOMENOLOGIA DAS RELAÇÕES SOCIAIS: MÉTODO, METODOLOGIA E DADOS RELEVANTES ACERCA DE PORTO ALEGRE E CANOAS}

Descrito o cenário no qual se encontra ambientada a investigação científica em curso, é chegado o momento de salientar que o objetivo mais relevante que a

de Bruxelles, Bruxelles, 2014. p. 66-67.

19 MARTINS, Fernando Rodrigues. Informação, sociedade em rede e situação jurídica subjetiva existencial. In SALOMÃO, José Fernando; BELTRÃO, Silvio Romero (Coord.). Direito civil: estudos em homenagem a José de Oliveira Ascensão. v. 1. São Paulo: Atlas, 2015. p. 311.

20 MARTÍN, Carlos Tapia. Espacios negativos: contra e anti como partículas reveladoras en el espacio. In RODRIGUEZ, José Exequiel Basini et al (Org.). Cidades, fronteiras e mobilidade humana. Manaus: EDUA, 2014. p. 125. "[El] pensamiento arquitectónico y condiciones de frontera pueden ser reunidos desde muy distintos posicionamientos, desde los tecnológi$\cos [\ldots]$, infraestructurales [...], hasta los disciplinares". É factível defender que dimensões jurídicas e sociais também devam ser aportadas à reflexão.

21 HUMANES, Mariano Pérez. Fronteras urbanas: el espacio público como espacio de recusión. In RODRIGUEZ, José Exequiel Basini et al (Org.). Cidades, fronteiras e mobilidade humana. Manaus: EDUA, 2014. p. 174. "Legisladores y arquitectos van de la mano de estas acciones donde la separación y la diferenciación de la población se lleva a cabo cerrando espacios, trazando lindes y fronteras". 
informa foi pinçado na necessidade de averiguar os problemas provocados com a difusão dos sistemas de videovigilância, em especial, quando se tem em mente que a liberdade e a igualdade, constitucionalmente prometidas no Brasil, são princípios com normatividade tão pulsante, quanto cogente.

A pesquisa foi pensada e executada em duas etapas.

Inicialmente, foram realizadas entrevistas buscando entender as razões que justificam e informam o investimento exigido na fase de implantação das redes de vigilância e controle ${ }^{22}$. A abordagem empírica revelou-se - ao menos, aparentemente - como o único meio que permitiria mapear, por exemplo, (a) como pensam os gestores, (b) o que orienta suas ações, (c) que preconceitos obnubilam sua percepção de mundo ou, ainda, (d) se há alguma preocupação com a proteção dos dados capturados pelas câmeras que se espalham pelas cidades de Porto Alegre e de Canoas, cidades eleitas não apenas porque são precursoras, no Rio Grande do Sul, na utilização das ferramentas tecnológicas referidas ao longo deste texto, mas também, por figurarem entre as maiores cidades do estado. Ademais, os objetivos delineados nesta investigação científica apontam para a desnecessidade de estender o mapeamento empírico a outras plagas. Relevante salientar, também, que ambas as cidades eleitas possuem (a) orçamentos volumosos, (b) população bastante grande e (c) níveis de violência não apenas elevados, mas em crescimento ${ }^{23}$.

\section{Foto 01}

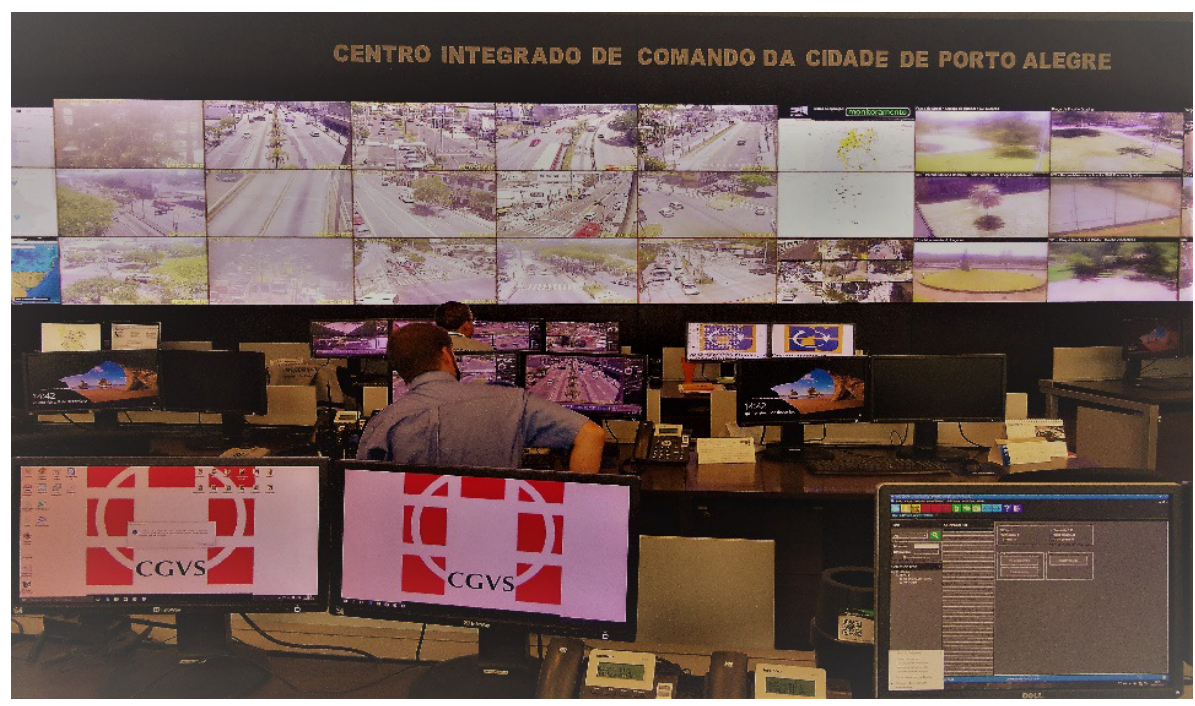

Créditos: Sara Dotto.

22 Saliente-se que, em ambos os casos, fomos bastante bem-recebidos.

23 SECRETARIA DE SEGURANÇA PÚBLICA. Indicadores Criminais, 2017. Disponível em: em <http://www.ssp.rs.gov.br/indicadores-criminais>. Acesso em 11.dez.2017. 
Uma vez obtidos os dados empíricos - por ocasião da segunda etapa deste trabalho -, foram encontrados, na literatura jurídica e não jurídica, os subsídios e elementos necessários para a reflexão e crítica alinhavados ao longo de todo o texto.

Rasgando alguns véus - dentre eles, os que obnubilam a ignorância que move esta pena -, o levantamento empírico realizado levou a identificar que, em ambas as cidades, (a) a principal justificativa para o investimento substancial realizado encontra-se intimamente fundida às promessas de ampliação dos níveis de segurança pública, embora (b) não tenha sido revelado, durante as entrevistas, que qualquer caso impactante - ideia aqui significada como a prática de crime de elevado potencial ofensivo - tenha sido desvendado com o apoio dos sistemas tecnológicos.

Em ambos os casos, foi narrado, ainda, por ocasião do contato empírico, que (c) os sistemas funcionam vinte e quatro horas por dia, sete dias por semana e que (d) os seus operadores, muitos dos quais, policiais aposentados, na reserva e (ou) vigilantes municipais, têm seus olhos treinados, em muitas situações, adestrados ao largo de toda uma vida dedicada ao trabalho no policiamento ostensivo, para identificar quaisquer situações de risco, (e) atuam entre oito e doze horas por dia, às vezes, diante de uma tela de computador com 16 quadros distintos na qual imagens transitam de forma incessantemente repetitiva.

\section{Foto 02}

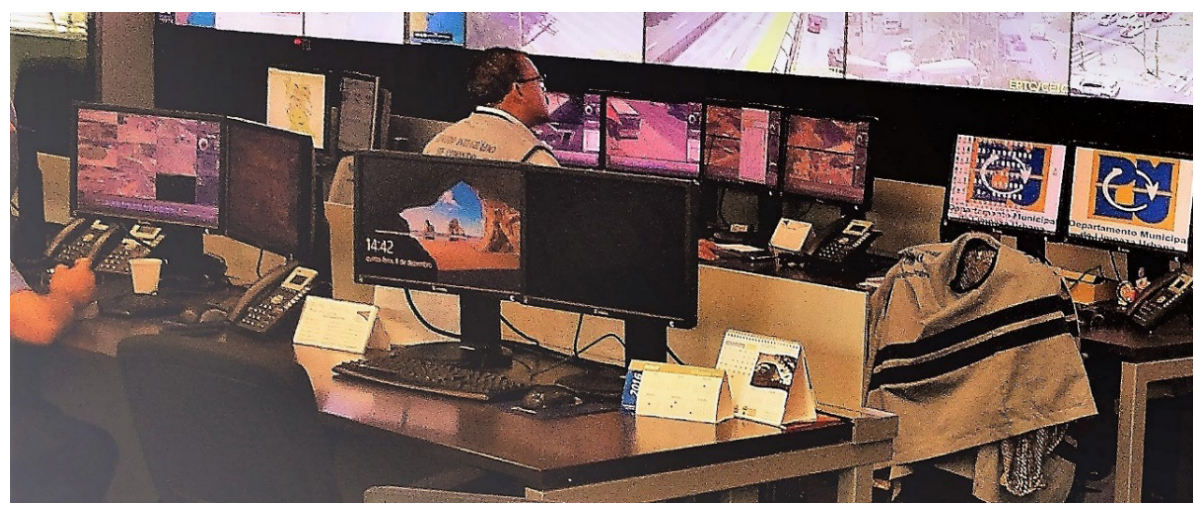

Créditos: Sara Dotto

Em ambas as entrevistas, também e com incomum naturalidade, (f) foram relatados o clamor da população e o interesse da municipalidade na ampliação dos sistemas por meio da instalação de mais câmeras e (ou) mais integração entre os agentes e (ou) secretarias municiadas pelas informações que fluem através dos olhos dos anjos, portanto, através das lentes das câmeras espalhadas pela urbe.

Outras coincidências foram identificadas a partir do material colhido durante as entrevistas realizadas. Coincidências que emergem no silêncio lan- 
çado por palavras não pronunciadas, pois, em ambas as entrevistas, não foram revelados (g) a quantidade de pessoas filmadas diariamente ou (h) o seu perfil, (i) qual seria o custo total dos investimentos realizados nos sistemas de videovigilância, tampouco (j) o custo mensal, para os cofres públicos, da manutenção dos centros de controle.

Além disso, enquanto, na visita realizada ao CCTV existente em Porto Alegre, (k) não foi autorizado acesso aos mapas contendo informações acerca da alocação das câmeras - sob a justificativa de que "os bandidos" não poderiam saber onde elas estão -, na cidade de Canoas (1) foi relatado, durante a entrevista, o desejo de estender a rede de videovigilância até os portões de acesso à cidade e, paralelamente, o de recorrer à tecnologia apta a promover a identificação das placas dos veículos que, através deles, ingressam e afastam-se da cidade. A entrevista havida em Canoas permitiu, ainda, $(\mathrm{m})$ aferir a existência de câmeras capturando imagens no interior de casas espalhadas pela cidade, prática que encontraria a sua justificativa na imperiosidade de combate à dengue e na desídia de um número incerto de proprietários.

Em momento algum, emergiram preocupações como as contidas neste estudo. Nenhuma palavra acerca de liberdades violadas ou tangenciando preocupações com a construção ou com o alargamento de estereótipos e a consequente negação da igualdade substancial. E, ainda que (n) o processo que permite acesso às imagens gravadas a eventuais interessados possua algumas travas de segurança - como a necessidade de requerimentos formais -, não foram relatadas quaisquer práticas que promovam, por exemplo, a desidentificação das pessoas capturadas nas cenas registradas ou com a ação potencial de hackers ou crakers, que poderiam ter acesso às ondas e (ou) cabos pelos quais as imagens transitam dos pontos de captura até os centros de controle.

A tabela abaixo talvez apresente outros dados úteis à reflexão ao concentrar informações referentes à população, ao orçamento anual, à importância estratégica de Porto Alegre e de Canoas no estado, o custo por câmera e o custo inicial do sistema, a quantidade de câmeras geridas em cada município, a relação existente entre o número de habitantes por câmera instalada, o número de ocorrências registradas, o sistema de transmissão de dados utilizado e a existência (ou não) de tecnologia de reconhecimento facial, o nível de zoom e, enfim, uma descrição sintética de como são armazenadas as imagens e como podem ser acessadas.

\section{Tabela 01 - Videovigilância em Canoas e Porto Alegre em números}

\begin{tabular}{ccc}
\hline Cidade & Porto Alegre & Canoas \\
\hline População & $1.409 .000(2010)$ & $323.827(2010)$
\end{tabular}

Orçamento anual do município

$\mathrm{R} \$ 6.949 .142 .987,00$ (2017) $\quad \mathrm{R} \$ 1.700 .417 .940,00$ (2017) 
Importância no Rio

Grande do Sul

Custo por câmera ${ }^{24}$

Custo inicial do

sistema

Câmeras geridas pelo

poder público

Relação habitantes

por câmera

Número de

ocorrências

registradas

Sistema de

transmissão de dados

Tecnologia de

reconhecimento

facial

Zoom

Pessoas filmadas

diariamente

Perfil das pessoas

filmadas ${ }^{28}$

Armazenamento das imagens

Acesso às imagens fazê-lo. Exige-se autorização judicial. $4^{\circ}$

$\mathrm{R} \$ 20.000 .00$

$\mathrm{R} \$ 8.000 .000,00^{25}$

186

$1400(2015)^{26}$

Rádio e cabo ótico.

Sob estudo.

250 metros

Há gravação e o registro é mantido por 30 dias.

Qualquer interessado pode fazê-lo dirigindo-se ao respectivo Centro de Atendimento.

Fonte: autoria própria.

24 Câmeras mais antigas podem ter sido adquiridas, por menor valor, em reais.

25 Dados referentes ao último aporte substancial. Não foram informados o valor de investimentos anteriores ou o custo mensal de manutenção do sistema de videovigilância.

26 Inexistem relatos de quantas podem ser consideradas crimes ou crimes com elevado potencial ofensivo.

27 Por conta dos custos elevados de acesso ao software.

28 Perguntou-se que espécie de imagem chama mais a atenção dos operadores e quem chama mais a atenção do observador? As perguntas não foram respondidas. 
Ela traz também - especialmente, naquilo que silencia, que parece não querer revelar ao mundo -, questionamentos deveras relevantes que, ao lado dos dados obtidos, dão vida a muitas das reflexões propostas ao largo desta investigação cientifica.

\section{A VINGANÇA DE ARGOS PANOPTES}

O mito de Argos Panoptes chegou à contemporaneidade modelado na forma de um gigante com cem olhos e que, exatamente por conta dessa característica, mesmo ao dormir, era capaz de manter a metade deles abertos. Morto por Hermes - antes que a mitologia deixasse de balizar boa parte das condutas humanas - ele ressurge agora entremeio o caos que impulsiona as cidades contemporâneas, não mais como mito e, sem dúvida, com muitos mais olhos que outrora ${ }^{29}$.

Olhos que são capazes de registrarem o passado e, consoante muitos creem, domesticarem o presente e corrigirem o futuro ${ }^{30}$ e que, espalhados - de modo nem sempre perceptível - pela urbe, legitimam a difusão da violência ${ }^{31}$ (a) potencializando a criação de estereótipos, (b) estimulando o voyeurismo, (c) facilitando a captura e registro de importantes momentos da vida íntima e, ainda, (d) auxiliando o processo de difusão da cultura do medo. Olhos que, portanto, uma vez fundidos à arquitetura jurídica da falsa legalidade ${ }^{32}$, amplificam o des-

29 LIPOVETSKY, Gilles. Los tiempos hipermodernos. Trad. Antonio Prometeo Moya. Barcelona: Anagrama, 2014. p. 57-58.

30 MELGAÇO, Lucas; VERFAILlIE, Kristof; HILDEBRANDT, Mireille. CCTV and Smart CCTV effectiveness: a meta-level analysis. SIAM - Security Impact Assessment Measures. Brussel: Vrije Universiteit Brussel, 2013. p. 12.

31 CARR, Robert. Political economy \& the abbott government's CCTV programme in Australia, University of New South Wales: unpublished manuscript. Not available anymore apud DEBAILLEUL Corentin; KEERSMAECKER Pauline De. Towards the panoptic city: the proliferation of video surveillance in Brussels and Copenhagen. Thesis (Master) - Université Libre de Bruxelles, Bruxelles, 2014. p. 12. "I have rationalised CCTV as: a component in the cultivation of patron-client ties between the Federal Government, its agents (MPs) and local councils/communities; a mechanism for government to facilitate the flow of public funds to private companies through arrangements that are virtually unchecked and non-evidence based; a mechanism for government to facilitate profitable opportunities for the security technologies industry, technical consultants and news media; and, a mechanism for reproducing political and social hegemony by working to normalise these relations at the level of discourse".

32 RODRIGUEZ, José Rodrigo. As figuras da perversão do direito: para um modelo crítico de pesquisa jurídica empírica, Revista Prolegómenos Derechos y Valores, v. 19, n. 37, 99-124, jan. / jun. 2016. p. 104-105. “O conceito de falsa legalidade é especialmente útil para evidenciar espaços de arbítrio no interior do estado de direito, espaços que passariam despercebidos se nos ativéssemos apenas ao texto das leis sem prestar atenção em sua aplicação e em seus efeitos sobre a sociedade. Sustento que uma das tarefas centrais da pesquisa empírica em direito hoje é, justamente, identificar casos de falsa legalidade para manter a legitimidade das promessas da democracia encarnadas na racionalidade do estado de direito". 
respeito a múltiplas dimensões da vida privada ${ }^{33}$, desnudando-a ${ }^{34}$.

Ademais, embora o silêncio propagado de forma eloquente pelos dados capturados no mapeamento empírico realizado não permita dizê-lo com tamanha precisão, assusta imaginar que, em alguma medida, as situações vividas no Brasil possam assemelhar-se aos delineados abaixo.

Eric Heilmann reviewed a 1999 study detailing the practices of CCTV operators in three different cities for 600 hours. It shows that prime targets of the operators are the teenagers, representing $47 \%$ of the targeted persons but only $15 \%$ of the local population. Men are more often targeted than women $(89 \%$ vs. $11 \%)$, and in a prosperous provincial town where 'black' people compose $6 \%$ of the population, although $15 \%$ of CCTV targeting is aimed at them. Among all the people specifically monitored by the operators, $30 \%$ were suspected (rightly or wrongly) to be involved in some kind of crime or offence, and $22 \%$ suspected to disrupt public order. For all the others, "no obvious reason" could justify their monitoring. Voyeurism represented $1 \%$ of the cases generally and $10 \%$ when it came to monitoring women ${ }^{35}$.

E, mesmo que a tais redes se possa atribuir papel relevante, ao se identificar que "as presented, video surveillance, whether traditional or "smart", functions through a series of mechanisms that can be classified into three main effects: "past", "present" and "future". The "past" effect, that is to say, the capacity of video surveillance to serve investigation purposes appeared as the one in which both CCTV and Smart CCTV are more effective in reducing crime" "36, é preciso notar, no vazio no qual se encontram alocados dados desconhecidos - e (ou) não relevados -, que, como comprovado em outras pesquisas tangenciando o tema aqui explorado, "the main result of the study is that no serious decrease in crime can be attributed to CCTV"37 -, o que pode ocorrer, também, porque "watching

33 LA RONCIÈRE, Charles de. A vida privada dos notáveis toscanos no limiar da Renascença. In DUBY, Georges (Org.). História da vida privada: da Europa feudal à Renascença. Trad. Maria Lúcia Machado. São Paulo: Companhia das Letras, 2009. p. 173. "Vizinhos: a palavra completa sempre a trilogia padrão (parentes, amigos, vizinhos) dos memorialistas. De fato, os vizinhos desempenham na vida cotidiana privada um papel que não é muito diferente daquele dos amigos e parentes, papel ao qual sua proximidade os predispõe. Não se esconde grande coisa de seus vizinhos. Eles têm mil ocasiões de intervir na vida cotidiana [...]".

34 AGAMBEN, Giorgio. Homo sacer: o poder soberano e a vida nua. Trad. Henrique Burigo. Belo Horizonte: UFMG, 2010.

35 DEBAILLEUL Corentin; KEERSMAECKER Pauline De. Towards the panoptic city: the proliferation of video surveillance in Brussels and Copenhagen. Thesis (Master) - Université Libre de Bruxelles, Bruxelles, 2014. p. 28.

36 MELGAÇO, Lucas; VERFAILliE, Kristof; HILDEBRANDT, Mireille. CCTV and Smart CCTV effectiveness: a meta-level analysis. SIAM - Security Impact Assessment Measures. Brussel: Vrije Universiteit Brussel, 2013. p. 28. Segue o autor afirmando que "[in] regards to the "present" function, that of detecting crimes in real time and acting upon it, CCTV seems to be very ineffective, whilst Smart CCTV showed better results. Concerning the "future" aspect, that is, deterrence, video surveillance appeared more effective in the first year after the installation of cameras. However, such effectiveness tends to decrease over time".

37 DEBAILLEUL Corentin; KEERSMAECKER Pauline De. Towards the panoptic city: the pro- 
video surveillance can be tedious and boring [and] often there can be hours or days of video from multiple sources where very little of interest actually happens" ${ }^{38}$. Tais constatações impõem, no mínimo, ponderar se a ação política e os investimentos públicos correlatos e necessários a sua implementação e execução - em especial, diante de seu potencial deletério no que toca à liberdade e à igualdade constitucionalmente garantidas no Brasil - não necessitam ser repensados, mormente, por conta (a) da pouca utilidade do processo de captura, registro e tratamento das imagens que atravessam as muitas íris vítreas das câmeras espalhadas pelas cidades ${ }^{39}$ e da correlata (b) ausência de níveis minimamente razoáveis de materialização das promessas que informam e tentam justificar a utilização das redes de videovigilância, (c) o seu custo econômico - são vinte mil reais por câmera e a esse valor devem ser acrescidos os custos mensais de energia elétrica, de manutenção de todo o equipamento, os custos de ocupação de espaços os sistemas de vigilância serão alocados, os salários de inúmeros servidores etc. -, bem como, diante do aparente $(\mathrm{d})$ desrespeito a direitos fundamentais ${ }^{40}$ envoltos, encobertos e obnubilados pela nevoa retórica que busca legitimar socialmente a troca, não negociada, de liberdades pela dominação high tech.

Aliás, no que toca, pontualmente, às questões concernentes à fundamentalidade de direitos atravessados pelas práticas catalogadas nessa investigação há de salientar-se que entremeio o início da pesquisa e a sua publicação ${ }^{41}$ veio ao mundo, em agosto de 2018, a Lei 13.709. A referida lei, cuja vacatio legis foi inicialmente projetada para 18 meses e, ulteriormente alterada ${ }^{42}$ para viger em parte, a partir do último 28 de dezembro e, em parte, vinte e quatro meses após a sua publicação, tem por objeto a proteção de dados pessoais, buscando aclarar,

liferation of video surveillance in Brussels and Copenhagen. Thesis (Master) - Université Libre de Bruxelles, Bruxelles, 2014. p. 22.

38 FERENBOK, J; CLEMENT, A. Hidden changes: from CCTV to "smart" video surveillance. In DOYLE, A.; LIPPERT, R; LYON, D. (Eds.). Eyes everywhere: the global growth of camera surveillance. New York: Routledgem 2012 apud MELGAÇO, Lucas; VERFAILLIE, Kristof; HILDEBRANDT, Mireille. CCTV and Smart CCTV effectiveness: a meta-level analysis. SIAM - Security Impact Assessment Measures. Brussel: Vrije Universiteit Brussel, 2013.

39 AGOSTINI, Aldo. Videosorveglianza e privacy. Bologna: Edis, 2006. p. 17-20.

40 Na impossibilidade de comprová-lo empiricamente, recorre-se a dados produzidos na Europa. Vide: PAISSAN, Mauro. La privacy è morta, viva la privacy. Milano: Ponte alle Grazie, 2009. p. 10. "Altro fronte delicato, la videosorveglianza. Le nostre città sono piene di telecamere, poste in luoghi a rischio di reati, ma anche davanti a palazzi, su pianerottoli condominiali, in giardini pubblici non così pericolosi. Le telecamere possono talvolta essere effetivamente utili a dissuadere i male intenzionati o a individuare responsibili di reati. Ma la loro proliferazione a dismisura rischia di essere controproducente. Una clamorosa conferma viene dalla Gran Bretagna, che detiene il record di videocamere, più di quattro milioni. [...] Abbiamo speso miliardi di sterline, ma solo nel ter per cento dei casi di furti e rapine in strada i responsabili sono stati catturati grazzie ale telecamere".

41 Fica aqui o registro de que a minudente revisão realizada no sistema double blind review foi a responsável por nos chamar a atenção para a lacuna ora preenchida com essas singelíssimas notas acerca da Lei Geral de Proteção de Dados Pessoais.

42 Nos termos da redação que lhe foi dada pela Medida Provisória n. 869/2018. 
desde seus primeiros momentos, que foca a tutela dos direitos fundamentais de liberdade e de privacidade e, ainda, o livre desenvolvimento da personalidade humana.

Anote-se, desde cedo, que a exclusão prevista no inciso III, alínea (a) do artigo $4^{\circ}$ da lei em questão ${ }^{43}$ não tem o condão de afastar a incidência das regras e princípios que informam ou informarão, em breve - nos termos dos artigos $7^{\circ}$ a 16 e 23 a 32 - o tratamento de dados pessoais nos cenários tocados por essa pesquisa o que ocorre, especialmente, por conta da multiplicidade da pluralidade de objetos alcançada pelos sistemas de videovigilância que buscam tratar, em algum nível, (a) as informações sobre tráfego e trânsito, (b) passeatas, (c) espetáculos e shows a céu aberto, (d) comércio ambulante, além de (e) cuidar da saúde e do bem-estar das pessoas que caminham por ruas e praças e (f) combater à dengue, além, obviamente, $(\mathrm{g})$ da promessa de cidades mais seguras.

É relevante trazer, uma vez mais, à tona a constatação de que [when] utilized for security purposes, surveillance cameras can be broadly classified according to three principal mechanisms, which can be explained in terms of the "past", "present" and "future" functions of the criminal activity. In relation to "past", cameras have the intention to record events and serve as a data bank for investigation and later identification of the criminal. The images can also be used in court as evidence. In the relation to the "present" function, the camera has the aim to serve as an extension of the eyes of the police or private security guards. The agent behind the cameras identifies a suspected activity or a crime already in the process of being committed and acts in real time, preventing it from being accomplished. The third goal, which turns to "future" time, refers to the capacity of the camera to prevent a crime from occurring by inducing a sensation into the criminal that he is being continuously monitored. Thus, a running camera, when connected to a system of data storage, responds to "past", "present" and "future" purposes as described. In other words, it permits investigation, detection and deterrence. However, a connected camera, that does not store images, only meets "present" and "future" functions. And finally, a false camera in which images are neither produced nor stored, has only a function in the "future", since its sole purpose is to induce the feeling of being monitored ${ }^{44}$.

Inúteis ou quase, aos fins aos quais se destinam, paradoxalmente, sistemas de videovigilância cada vez mais inteligentes ${ }^{45}$ - e, talvez, cada vez mais caros, não foi possível identificar - espalham-se pelas cidades ampliando, em vez de evitá-los, riscos das mais distintas ordens.

43 Art. $4^{\circ}$. Esta Lei não se aplica ao tratamento de dados pessoais: [...] III - realizado para fins exclusivos de: (a) segurança pública.

44 MElgaÇO, Lucas; VERFAILlie, Kristof; HILDEBRANDT, Mireille. CCTV and Smart CCTV effectiveness: a meta-level analysis. SIAM - Security Impact Assessment Measures. Brussel: Vrije Universiteit Brussel, 2013. p. 12.

45 Idem. p. 20. "Detection, tracking and classification of targets are the main tasks expected from video analytics engines. Detection refers to the identification of what physical objects exist in the surveillance area, tracking is the understanding of how they move and classification is related to the labelling of objects as human, vehicle, animal and to the interpretation as normal or abnormal objects or behaviours". 
Riscos como (a) os esboçados na possibilidade, narrada na entrevista realizada na cidade de Canoas, de uma pessoa vir a ser filmada dentro de um espaço que deveria ser o último bastião na salvaguarda do ser humano ${ }^{46}$ contra os desmandos de um Príncipe que se acreditava ter sido deposto com o surgimento das constituições da Modernidade ou - dentre tantos outros ignorados aqui - que se encontram (b) atados ao "abuso de la información, bien sea por compartir los datos sin autorización, por integración de bases de datos por compañias para desarrollar un perfil extremadamente detallado de una persona o por su divulgación y utilización no autorizada"47.

Enquanto isso, o Mercado reina soberano ${ }^{48}$, alimentando-se de modo pantagruélico ${ }^{49}$.

\section{CONCLUSÕES INCONCLUSIVAS: SOBRE SOMBRAS E VAGA- LUMES}

As regras que versam sobre proteção de dados - mesmo nos países nos quais existem ${ }^{50}$ - parecem não resolver problemas não percebidos por olhos cansados, domesticados ou que teimam em não enxergar que a tutela da intimidade emerge da rebelião do indivíduo contra a opressão moralizadora que, por longa data,

46 CF/88. "Art. $5^{\circ}$ Todos são iguais perante a lei, sem distinção de qualquer natureza, garantindo-se aos brasileiros e aos estrangeiros residentes no País a inviolabilidade do direito à vida, à liberdade, à igualdade, à segurança e à propriedade, nos termos seguintes: [...] X - são invioláveis a intimidade, a vida privada, a honra e a imagem das pessoas, assegurado o direito a indenização pelo dano material ou moral decorrente de sua violação. XI - a casa é asilo inviolável do indivíduo, ninguém nela podendo penetrar sem consentimento do morador, salvo em caso de flagrante delito ou desastre, ou para prestar socorro, ou, durante o dia, por determinação judicial”.

47 SANCLEMENTE, María del Rosario Rivera. La evolución de las estrategias de marketing en el entorno digital: implicaciones jurídicas. Tesis (Doctorado) - Universidad Carlos III, Madrid, 2016. p. 127.

48 AGAMBEN, Giorgio. Homo sacer: o poder soberano e a vida nua. Trad. Henrique Burigo. Belo Horizonte: UFMG, 2010. p. 38. “O soberano é o ponto de indiferença entre violência e direito".

49 BÉTIN, Christophe; MARTINAIS, Emmanuel; RENARD, Marie-Christine. Sécurité, vidéosurveillance et construction de la déviance: l'exemple du centre ville de Lyon, Déviance et Société, v. 27, n. 1, p. 3-24, 2003 apud DEBAILLEUL Corentin; KEERSMAECKER Pauline De. Towards the panoptic city: the proliferation of video surveillance in Brussels and Copenhagen. Thesis (Master) - Université Libre de Bruxelles, Bruxelles, 2014. p. 16. "We can indeed assess that when it comes to justifying the installation of an urban CCTV system, there is a phenomenon of designation of some fringes of the population as being inherently harmful. Economic interests play an important role in the setting of CCTV systems, and they are often at stake in the definition of who is a legitimate citizen and who is a deviant one".

50 BERNABÈ, Franco. Libertà vigilata: privacy, sicurezza e mercato nella rete. Bari: Laterza, 2012. p. 46. "Rizervatezza e protezione dei datti personali sono spesso descritte in termini di gemelli, ma non identici. Contrariamente alle regole in materia di diritto alla riservatezza, le norme sulla protezione dei datti non sono inibitorie, non marcano cioè un divieto di utilizzo o di divulgazione di determinate informazioni, ma si limitano semplicemente a definere le regole (condizioni, modalità e autorizzazioni) che devono essere rispettate affinché l'utilizzo possa essere considerato legittimo". 
balizou o comportamento da humanidade ${ }^{51}$ ou que "el proceso de inmunización de la vida ciudadana ha sido un proceso de domesticación, normalización y artificialización progresivo, que ha ido acompañado de un proceso de privatización $y$ de homologación no solo de los espacios, sino de los modos de vida" ${ }^{2}$.

A ágora está sendo, lentamente, cercada. Entremeio a esse movimento, seus habitantes, lentamente, dia após dia, são privados de seus direitos. Talvez, sem perceber - embora, com esperada docilidade ${ }^{53}$, - eles têm suas liberdades, continua e suavemente, desbastadas, corroídas ou carcomidas nos mais distintos espaços de convivência urbana.

Eis, talvez, o momento de resgatar a aposta de Pascal ${ }^{54}$ : se liberdade e igualdade vencerem, excelente. Ganha a democracia, vence a fundamentalidade dos direitos civis. Se forem vencidas, nada será sacrificado, pois parece pouco factível acreditar - não sem refutação ${ }^{55}$ - que um mundo mais absurdo ${ }^{56}$ do que aquele no qual a humanidade se encontra imersa possa estar contido no porvir.

E, se é certo que não há um algoritmo conhecido que possa resolver o problema denunciado neste texto, parece igualmente defensável que o silêncio ecoa como a pior das escolhas. Mesmo que lançadas a esmo, cada mensagem "na garrafa é um testemunho da transitoriedade da frustração e da permanência da esperança, da indestrutibilidade das possibilidades e da fragilidade das adversidades que impedem sua implementação" ${ }^{57}$.

Quiçá, vagalumes voltem, em breve, a flanar pelos céus do Brasil ${ }^{58}$.

51 ROBL FILHO, Ilton Norberto. Direito, intimidade e vida privada: paradoxos jurídicos e sociais na sociedade pós-moralista e hipermoderna. Curitiba: Juruá, 2010. p. 58.

52 HUMANES, Mariano Pérez. Fronteras urbanas: el espacio público como espacio de recusión. In RODRIGUEZ, José Exequiel Basini et al (Org.). Cidades, fronteiras e mobilidade humana. Manaus: EDUA, 2014. p. 168.

53 AGAMBEN, Giorgio. Homo sacer: o poder soberano e a vida nua. Trad. Henrique Burigo. Belo Horizonte: UFMG, 2010. p. 11. "O desenvolvimento e o triunfo do capitalismo não teria sido possível [...] sem o controle disciplinar efetuado pelo novo biopoder, que criou para si, através de uma série de tecnologias apropriadas, os corpos dóceis de que necessitava".

54 PASCAL, Blaise. Pensamentos. Mem Martins: Europa-América, 1988.

55 HARARI, Yuval Noah. Homo Deus: uma breve história do amanhã. Trad. Paul Geiger. São Paulo: Companhia das Letras, 2016. p. 106. "Em anos recentes, quando se começou a repensar as relações entre humanos e animais, essas práticas [gaiolas, chiqueiros etc.] passaram a ser alvo de críticas crescentes. Demonstramos um interesse inédito no destino das chamadas formas inferiores de vida, talvez porque estejamos a ponto de nos tornar uma. Se e quando programas de computador atingirem uma inteligência sobre-humana e um poder jamais visto, deveremos valorizar esses programas mais do que valorizamos os humanos? Seria aceitável, por exemplo, que uma inteligência artificial explorasse os humanos e até os matasse para contemplar as necessidades de seus próprios desejos? Se a resposta [for] negativa, a despeito da inteligência e do poder superiores, porque é ético que os humanos explorem e matem porcos? Com efeito, o que há nos humanos que nos faz tão inteligentes e poderosos e qual é a probabilidade de que entidades não humanas venham a rivalizar conosco e nos ultrapassar?”

56 CAMUS, Albert. O mito de Sísifo. São Paulo: Record, 2004.

57 BAUMAN, Zygmunt. Para que serve a sociologia? Trad. Carlos Alberto Medeiros. Rio de Janeiro: Zahar, 2015. p. 54.

58 DIDI-HUBERMAN, Georges. Sobrevivência dos vaga-lumes. Trad. Vera Casa Nova e Márcia 


\section{REFERÊNCIAS}

AGAMBEN, Giorgio. Homo sacer: o poder soberano e a vida nua. Trad. Henrique Burigo. Belo Horizonte: UFMG, 2010.

AGOSTINI, Aldo. Videosorveglianza e privacy. Bologna: Edis, 2006.

ALVES, Manoel Rodrigues; RIZEK, Cibele Saliba. Cidade contemporânea, cidade do empresariamento: aspectos da produção socioespacial do urbano. In RODRIGUEZ, José Exequiel Basini et al (Org.). Cidades, fronteiras e mobilidade humana. Manaus: EDUA, 2014.

BAUMAN, Zygmunt. Para que serve a sociologia? Trad. Carlos Alberto Medeiros. Rio de Janeiro: Zahar, 2015.

BERNABÈ, Franco. Libertà vigilata: privacy, sicurezza e mercato nella rete. Bari: Laterza, 2012.

BÉTIN, Christophe; MARTINAIS, Emmanuel; RENARD, Marie-Christine. Sécurité, vidéosurveillance et construction de la déviance: l'exemple du centre ville de Lyon, Déviance et Société, v. 27, n. 1, p. 3-24, 2003.

BOURDOUX, Gil. Vidéosurveillance et police. Quel(s) coût(s) pour la police? Quel(s) coût(s) pour la société?. In DEVRESSE, Marie-Sophie; PIERET, Julien (Dir.). La vidéosurveillance: entre usages politiques et pratiques policières, Brussels: Politeia, 2009.

CAMUS, Albert. O mito de Sísifo. São Paulo: Record, 2004.

CARDARELLI, Francesco. Amministrazione digitale, trasparenza e principio di legalità. Il Diritto Dell'informazione e Dell'informatica, v. 31, n. 2, p. 227-273. mar. / apr. 2015.

CATALAN, Marcos. Avanço da técnica e afetação da vida privada no espaço público. Revista Direitos Humanos e Democracia, v. 6, p. 455-464, 2018.

CARR, Robert. Political economy \& the abbott government's CCTV programme in Australia, University of New South Wales: unpublished manuscript.

COSTA, Renata Almeida da. Cultura do medo e espaço urbano: um olhar reflexivo sobra a sensação social de insegurança. In SCHWARTZ, Germano; FERNÁNDEZ, Albert Noguera. (Org.). Cultura e identidade em tempo de transformações. Curitiba: Juruá. 2011.

DE'CARLI, Natália. La frontera del miedo global: proteger para ser. In RODRIGUEZ, José Exequiel Basini et al (Org.). Cidades, fronteiras e mobilidade humana. Manaus: EDUA, 2014. p. 158.

DEBAILLEUL Corentin; KEERSMAECKER Pauline De. Towards the panoptic city: the proliferation of video surveillance in Brussels and Copenhagen. Thesis (Master) - Université Libre de Bruxelles, Bruxelles, 2014.

Arbex. Belo Horizonte: UFMG, 2011. 
DIDI-HUBERMAN, Georges. Sobrevivência dos vaga-lumes. Trad. Vera Casa Nova e Márcia Arbex. Belo Horizonte: UFMG, 2011.

DUBY, Georges. A emergência do indivíduo: a solidão nos séculos XI-XIII. In DUBY, Georges (Org.). História da vida privada: da Europa feudal à Renascença. Trad. Maria Lúcia Machado. São Paulo: Companhia das Letras, 2009.

FAROCKI, Harun. Desconfiar en las imagenes. Buenos Aires: Caja Negra Editoria, 2013.

GOMEZ, Rakel Bozza. Em busca do habitar e do espaço do lugar. In RODRIGUEZ, José Exequiel Basini et al (Org.). Cidades, fronteiras e mobilidade humana. Manaus: EDUA, 2014.

GUEDES, Jefferson Carús. Dimensões linguísticas da desigualdade no Brasil: os diversos nomes legais de um mesmo fenômeno. Revista Brasileira de Políticas Públicas, v. 5, p. 59-76, 2015.

HARARI, Yuval Noah. Homo Deus: uma breve história do amanhã. Trad. Paul Geiger. São Paulo: Companhia das Letras, 2016.

HEMPEL, Leon; TÖPFER, Eric. CCTV in Europe. Final Report. UrbanEye project: on the threshold to urban Panopticon? Analysing the employment of CCTV in European cities and assessing its social and political impacts, Berlin: Centre for technology and society of the Technical University Berlin, 2004. p. 3-68. Disponível em: em <www.urbaneye.net/results/results.htm>. Acesso em 19.jan.2016.

HUMANES, Mariano Pérez. Fronteras urbanas: el espacio público como espacio de recusión. In RODRIGUEZ, José Exequiel Basini et al (Org.). Cidades, fronteiras e mobilidade humana. Manaus: EDUA, 2014.

JACOBSEN, Michael Hviid; TESTER, Keith. Introdução. In BAUMAN, Zygmunt. Para que serve a sociologia? Trad. Carlos Alberto Medeiros. Rio de Janeiro: Zahar, 2015. p. 13-14.

LA RONCIÈRE, Charles de. A vida privada dos notáveis toscanos no limiar da Renascença. In DUBY, Georges (Org.). História da vida privada: da Europa feudal à Renascença. Trad. Maria Lúcia Machado. São Paulo: Companhia das Letras, 2009.

LYON, David. L’occhio elettronico. Milano, Feltrinelli, 1997.

LYON, David. La società sorvegliata. Milano: Feltrinelli, 2002.

MARTíN, Carlos Tapia. Espacios negativos: contra e anti como partículas reveladoras en el espacio. In RODRIGUEZ, José Exequiel Basini et al (Org.). Cidades, fronteiras e mobilidade humana. Manaus: EDUA, 2014.

MARTINS, Fernando Rodrigues. Informação, sociedade em rede e situação jurídica subjetiva existencial. In SALOMÃO, José Fernando; BELTRÃO, Silvio 
Romero (Coord.). Direito civil: estudos em homenagem a José de Oliveira Ascensão. v. 1. São Paulo: Atlas, 2015.

MASI, Domenico de. A sociedade pós-industrial. 4. ed. Trad. Anna Maria Capovilla et all. São Paulo: Senac, 2003.

MELGAÇO, Lucas; VERFAILLIE, Kristof; HILDEBRANDT, Mireille. CCTV and Smart CCTV effectiveness: a meta-level analysis. SIAM - Security Impact Assessment Measures. Brussel: Vrije Universiteit Brussel, 2013.

MIRANDA, Victor Vasconcelos. O direito à privacidade na era digital e as tutelas assecuratórias. Revista Fórum de Direito Civil, Belo Horizonte, v. 5, n. 12, p. 97-121, maio/ago. 2016.

PAISSAN, Mauro. La privacy è morta, viva la privacy. Milano: Ponte alle Grazie, 2009.

PASCAL, Blaise. Pensamentos. Mem Martins: Europa-América, 1988.

ROBL FILHO, Ilton Norberto. Direito, intimidade e vida privada: paradoxos jurídicos e sociais na sociedade pós-moralista e hipermoderna. Curitiba: Juruá, 2010.

RODRIGUEZ, José Rodrigo. As figuras da perversão do direito: para um modelo crítico de pesquisa jurídica empírica, Revista Prolegómenos Derechos y Valores, v. 19, n. 37, 99-124, jan./jun. 2016.

SANCLEMENTE, María del Rosario Rivera. La evolución de las estrategias de marketing en el entorno digital: implicaciones jurídicas. Tesis (Doctorado) Universidad Carlos III, Madrid, 2016.

SECRETARIA DE SEGURANÇA PÚBLICA. Indicadores Criminais, 2017. Disponível em: em <http://www.ssp.rs.gov.br/indicadores-criminais>. Acesso em 11.dez.2017.

SUÁREZ, Carlo Emilio Piazzini. Los estudios socioespaciales: campo de tensiones y caminos recorridos. In RODRIGUEZ, José Exequiel Basini et al (Org.). Cidades, fronteiras e mobilidade humana. Manaus: EDUA, 2014.

XAVIER, Marilia Pedroso; XAVIER, Luciana Pedroso. A crescente violação do direito de imagem do cidadão diante do monitoramento das câmeras de vigilância. In AFORNALLI, Maria Cecília Naréssi Munhoz; GABARDO, Emerson (Coord.). Direito, informação e cultura: o desenvolvimento a partir de uma linguagem democrática. Belo Horizonte: Fórum, 2012.

ZICCARDI, Giovanni. Il computer e il giurista. Milano: Giuffrè, 2015.

Recebido em: 02/08/2018.

Aprovado em: 04/04/2019. 
